\title{
Chern numbers for two families of noncommutative Hopf fibrations ${ }^{1}$
}

\author{
Piotr M. Hajac ${ }^{a}$, Rainer Matthes ${ }^{b}$, Wojciech Szymański $^{c}$ \\ a Mathematisches Institut, Universität München, Theresienstr. 39, München, 80333, Germany; \\ and Instytut Matematyczny, Polska Akademia Nauk, ul. Śniadeckich 8, Warszawa, 00-950 \\ Poland; and Katedra Metod Matematycznych Fizyki, Uniwersytet Warszawski, ul. Hoża 74, \\ Warszawa, 00-682 Poland; URL: http://www.fuw.edu.pl/ ${ }^{\sim p m h}$ \\ ${ }^{b}$ Fachbereich 2, TU Clausthal, Leibnizstr. 4, D-38678 Clausthal-Zellerfeld, Germany; E-mail: \\ ptrm@pt.tu-clausthal.de \\ $c$ School of Mathematical and Physical Sciences, The University of Newcastle, Callaghan, NSW \\ 2308, Australia; E-mail: wojciech@frey.newcastle.edu.au
}

\begin{abstract}
.
We consider noncommutative line bundles associated with the Hopf fibrations of $S U_{q}(2)$ over all Podleś spheres and with a locally trivial Hopf fibration of $S_{p q}^{3}$. These bundles are given as finitely generated projective modules associated via 1-dimensional representations of $U(1)$ with Galois-type extensions encoding the principal fibrations of $S U_{q}(2)$ and $S_{p q}^{3}$. We show that the Chern numbers of these modules coincide with the winding numbers of representations defining them.
\end{abstract}

\section{Abridged version}

In this paper, we combine the algebraic tool of Galois-type extensions with the analytic tool of the noncommutative index formula to study two kinds of examples of quantum fibrations. Our main result is that the line bundles associated to these principal fibrations are mutually non-isomorphic. This gives an estimate of the positive cones of the algebraic $K_{0}$-groups of the base-space quantum spheres.

Let $B \subseteq P$ be an inclusion of algebras such that $B$ is the coinvariant subalgebra for some coalgebra $C$ coaction $\Delta_{R}: P \rightarrow P \otimes C$. Using the framework of Galois-type extensions, one can say when such an extension of algebras is principal. (The definition is tuned in such a way that for commutative algebras it coincides with the concept of affine group scheme torsors - the principal bundles of algebraic geometry.) Every principal $C$-extension $B \subseteq P$ allows one to assign to any finite-dimensional corepresentation of $C$ a finitely generated projective left $B$-module of colinear homomorphisms $\operatorname{Hom}^{C}\left(V_{\varphi}, P\right)[2]$. Taking its class in $K_{0}(B)$ and composing it with the Chern character defines the Chern-Galois character from the space of all finite-dimensional corepresentations of $C$ to the even cyclic homology of $B$. On the other hand, the $K$-homology Chern character assigns to finitely summable Fredholm modules cyclic cocycles. In the 1-summable case it takes a particularly simple form, notably it turns a pair of bounded $*$-representations $\left(\rho_{1}, \rho_{2}\right)$ into a trace (cyclic 0 -cocycle) on $B$ via the formula $\operatorname{tr}_{\rho}=\operatorname{Tr} \circ\left(\rho_{1}-\rho_{2}\right)$. The evaluation of this trace on the Chern-Galois character applied to a corepresentation gives a numerical invariant of the $K_{0^{-}}$ class of the module defined by this corepresentation. Moreover, for our examples, the integrality of these invariants (guaranteed by the noncommutative index formula) makes them computable.

\footnotetext{
${ }^{1}$ See the home page of P.M.H. for a preliminary detailed version of this work.
} 
Our first example of a noncommutative Hopf fibration has its source in Klimek-Lesniewski quantum discs and the idea of local triviality. Here we have a principal $\mathcal{O}(U(1))$-extension $\left.\mathcal{O}\left(S_{p q}^{2}\right) \subseteq \mathcal{O}\left(S_{p q}^{3}\right)\right)[8]$, and every 1-dimensional corepresentation $\varphi_{\mu}(1)=1 \otimes u^{-\mu}$ yields a finitely generated projective $\mathcal{O}\left(S_{p q}^{2}\right)$-module (quantum line bundle) $\mathcal{O}\left(S_{p q}^{3}\right)_{\mu}:=\operatorname{Hom}^{\mathcal{O}(U(1))}\left(\mathbb{C}_{\varphi_{\mu}}, \mathcal{O}\left(S_{p q}^{3}\right)\right.$ ). On the other hand, we have the following irreducible bounded $*$-representations of $\mathcal{O}\left(S_{p q}^{2}\right)[4]$ :

$$
\begin{aligned}
& \rho_{1}\left(f_{0}\right) e_{k}=\left(1-p^{k}\right) e_{k}, \quad \rho_{1}\left(f_{1}\right) e_{k}=\sqrt{1-p^{k+1}} e_{k+1}, \quad k \geq 0 \\
& \rho_{2}\left(f_{0}\right) e_{k}=e_{k}, \quad \rho_{2}\left(f_{1}\right) e_{k}=\sqrt{1-q^{k+1}} e_{k+1}, \quad k \geq 0 .
\end{aligned}
$$

Here $\left\{e_{k}\right\}_{k \geq 0}$ is an orthonormal basis of a separable Hilbert space and $f_{0}, f_{1}$ are generators of the *-algebra $\mathcal{O}\left(S_{p q}^{2}\right)$. These representations give a desired trace, and bring us to our first main result:

Theorem 0.1 For all $\mu \in \mathbb{Z}$, the pairing between the cyclic 0-cocycle $\operatorname{tr}_{\rho}$ and the $K_{0}$-class of $\mathcal{O}\left(S_{p q}^{3}\right)_{\mu}$ (Chern number) coincides with the winding number $\mu$, i.e., $\left\langle\operatorname{tr}_{\rho},\left[\mathcal{O}\left(S_{p q}^{3}\right)_{\mu}\right]\right\rangle=\mu$.

Our second example is a family of noncommutative Hopf fibrations of $S U_{q}(2)$ over all Podleś quantum spheres $S_{q, s}^{2}, s \in[0,1]$. As handling the generic Podleś spheres requires going beyond the Hopf-Galois theory, they were among main motivating examples driving the development of the theory of principal coalgebra extensions of noncommutative rings. Now, much as before, we associate to every corepresentation $\varphi_{\mu}$ a finitely generated projective left $\mathcal{O}\left(S_{q, s}^{2}\right)$-module $\mathcal{O}\left(S U_{q}(2)\right)_{\mu, s}:=\operatorname{Hom}^{\mathcal{O}\left(S U_{q}(2)\right) / J_{s}}\left(\mathbb{C}_{\varphi_{\mu}}, \mathcal{O}\left(S U_{q}(2)\right)\right)$. Here $J_{s}$ is the coideal right ideal generated by $K, L-s, L^{*}-s$, and $K, L$ generate the polynomial algebras $\mathcal{O}\left(S_{q, s}^{2}\right)$. One can show that $\mathcal{O}\left(S U_{q}(2)\right) / J_{s}$ coincides with $\mathcal{O}(U(1))$ viewed as a coalgebra, and that $\mathcal{O}\left(S_{q, s}^{2}\right) \subseteq \mathcal{O}\left(S U_{q}(2)\right)$ is a principal $\mathcal{O}\left(S U_{q}(2)\right) / J_{s}$-extension $[1,12,3]$. Next, the representations [13]

$$
\begin{gathered}
\pi_{-}(K) e_{n}=-s^{2} q^{2 n} e_{n}, \quad \pi_{-}(L) e_{n}=\lambda_{n}^{-}(q, s) e_{n-1}, \quad \lambda_{n}^{-}(q, s)=s \sqrt{1-\left(1-s^{2}\right) q^{2 n}-s^{2} q^{4 n}} \\
\pi_{+}(K) e_{n}=q^{2 n} e_{n}, \quad \pi_{+}(L) e_{n}=\lambda_{n}^{+}(q, s) e_{n-1}, \quad \lambda_{n}^{+}(q, s)=\sqrt{s^{2}+\left(1-s^{2}\right) q^{2 n}-q^{4 n}},
\end{gathered}
$$

form a needed 1-summable Fredholm module [11], and we have our second main result:

Theorem 0.2 For all $\mu \in \mathbb{Z}$, the pairing between the cyclic 0-cocycle $\operatorname{tr}_{\pi}$ and the $K_{0}$-class of $\mathcal{O}\left(S U_{q}(2)\right)_{\mu, s}$ (Chern number) coincides with the winding number $\mu$, i.e., $\left\langle\operatorname{tr}_{\pi},\left[\mathcal{O}\left(S U_{q}(2)\right)_{\mu, s}\right]\right\rangle=\mu$.

\section{Introduction}

Herein we study two families of noncommutative deformations of the Hopf fibration $S^{3} \rightarrow S^{2}$. The first one is based on the idea of local triviality. We can view $S^{2}$ as the gluing of two discs along their boundaries, and $S^{3}$ as the gluing of two solid tori along their boundaries (a Heegaard splitting of $S^{3}$ ). Then the classical discs can be replaced by Klimek-Lesniewski quantum discs [9], and subsequently a two-parameter family of noncommutative Hopf fibrations can be constructed $[5,8]$. The other family originates from the theory of quantum groups. First, one considers $S^{3}$ as $S U(2)$ and deforms it into the quantum group $S U_{q}(2)$ [14]. Then the classification of $S U_{q}(2)$ quantum homogeneous spaces yields a two-parameter family of noncommutative two-spheres [13]. The latter form the base of the Hopf fibrations of $S U_{q}(2)$ [3].

Function algebras of total spaces of principal $U(1)$-bundles always decompose into direct sums of sections of all associated line bundles. The same phenomenon occurs for both of the aforementioned deformations, i.e., the coordinate algebras $\mathcal{O}\left(S_{p q}^{3}\right)$ and $\mathcal{O}\left(S U_{q}(2)\right)$ are direct sums of associated finitely generated projective modules. The aim of this paper is to prove that these modules are mutually non-isomorphic. 
To achieve this, we take an advantage of the K-homology Chern character [6] and the ChernGalois character [2]. The former produces cyclic cocycles out of bounded representations of the base algebra, and the latter cyclic cycles out of finite dimensional corepresentations of the structure coalgebra that define associated modules. The evaluation of these cocycles on these cycles gives $K_{0}$-invariants of the modules. The noncommutative index formula [6] shows that these invariants are indices of Fredholm operators, so that they have to be integers. This fact is essentially used in carrying out the computations.

Throughout this paper we work with unital algebras over a field (complex numbers in the studied examples) and adopt the standard Hopf-algebraic notation $m, \Delta, \varepsilon, S$ for the multiplication, comultiplication, counit and antipode, respectively. The symbolic notation $\mathcal{O}$ (quantum space) means a polynomial algebra defined by generators and relations, and $\mathrm{Tr}$ stands for the operator trace.

\section{Principal extensions and summable Fredholm modules}

Let $C$ be a coalgebra and $P$ an algebra and a right $C$-comodule via $\Delta_{R}: P \rightarrow P \otimes C$. Put $B=P^{c o C}:=\left\{b \in P \mid \Delta_{R}(b p)=b \Delta_{R}(p), \forall p \in P\right\}$. We say that the inclusion $B \subseteq P$ is a $C$-extension. A $C$-extension $B \subseteq P$ is called principal [2] iff

1. the Galois map $P \otimes_{B} P \stackrel{\text { can }}{\longrightarrow} P \otimes C, p \otimes p^{\prime} \mapsto p \Delta_{R}\left(p^{\prime}\right)$ is bijective;

2. there exists a left $B$-linear right $C$-colinear splitting of the multiplication map $B \otimes P \rightarrow P$;

3. the canonical entwining map $C \otimes P \stackrel{\psi}{\rightarrow} P \otimes C, c \otimes p \mapsto \operatorname{can}\left(\operatorname{can}^{-1}(1 \otimes c) p\right)$ is bijective;

4. there is a group-like $e \in C$ such that $\Delta_{R}(p)=\psi(e \otimes p), \forall p \in P$.

In order to define a strong connection on a principal extension, first we need to define a bicomodule structure on $P \otimes P$. The tensor product $P \otimes P$ is a right $C$-comodule via $\Delta_{R}^{\otimes}:=$ id $\otimes \Delta_{R}$. Since $\psi$ is bijective, it is also a left $C$-comodule via $\Delta_{L}^{\otimes}:=\left(\psi^{-1} \circ(\right.$ id $\left.\otimes e)\right) \otimes$ id. The two coactions evidently commute. Now, let $\pi_{B}: P \otimes P \rightarrow P \otimes_{B} P$ be the canonical surjection. A linear map $\ell: C \rightarrow P \otimes P$ is called a strong connection [2] iff it satisfies can $\circ \pi_{B} \circ \ell=1 \otimes \mathrm{id}$, $\Delta_{R}^{\otimes} \circ \ell=(\ell \otimes \mathrm{id}) \circ \Delta, \Delta_{L}^{\otimes} \circ \ell=(\mathrm{id} \otimes \ell) \circ \Delta$, and $\ell(e)=1 \otimes 1$. If $B \subseteq P$ is a principal $C$-extension and $\varphi: V_{\varphi} \rightarrow V_{\varphi} \otimes C$ is a finite dimensional corepresentation, then the left $B$-module $\operatorname{Hom}^{C}\left(V_{\varphi}, P\right)$ of all colinear maps from $V_{\varphi}$ to $P$ is finitely generated projective [2]. Denote by $\operatorname{Corep}_{f}(C)$ the space of all finite-dimensional corepresentations of $C$. Then a principal $C$-extension $B \subseteq P$ yields a map $\varphi \mapsto\left[\operatorname{Hom}^{C}\left(V_{\varphi}, P\right)\right]$ from $\operatorname{Corep}_{f}(C)$ to $K_{0}(B)$. The composition of this map with the Chern character $K_{0}(B) \rightarrow H C_{\text {even }}(B)$ (e.g., see [10]) is called the Chern-Galois character [2] and is denoted by chg. Explicitly, in degree 0, we have $\operatorname{ch} g_{0}(\varphi)=\left[c_{\varphi}^{<2>} c_{\varphi}^{<1>}\right]$. Here $c_{\varphi}$ is the character of $\varphi$, i.e., $c_{\varphi}=\sum_{i=1}^{\operatorname{dim} V} e_{i i}, \varphi\left(e_{j}\right)=\sum_{i=1}^{\operatorname{dim} V} e_{i} \otimes e_{i j},\left\{e_{i}\right\}$ a basis of $V_{\varphi}$, and $\ell(c)=: c^{<1>} \otimes c^{<2>}$ (summation understood).

Let us now recall the analytic tool of Fredholm modules that are used in the sequel. A $p$ summable Fredholm module over a $*$-algebra $B$ can be viewed as a pair $\left(\rho_{1}, \rho_{2}\right)$ of bounded *representations of $B$ such that $\operatorname{Tr}\left|\rho_{1}(b)-\rho_{2}(b)\right|^{p}<\infty$ for all $b \in B$ (see [6, p.88] for related details). The $K$-homology Chern character assigns to finitely summable Fredholm modules cyclic cocycles [6]. For $p=1$ it takes a particularly simple form, notably it turns $\left(\rho_{1}, \rho_{2}\right)$ into a trace (cyclic 0 -cocycle) on $B$ via the formula $\operatorname{tr}_{\rho}(b)=\operatorname{Tr}\left(\rho_{1}(b)-\rho_{2}(b)\right)$. 


\section{A locally trivial quantum Hopf bundle}

Let us consider the two-parameter family [5] of $*$-algebras $\mathcal{O}\left(S_{p q}^{3}\right), 0 \leq p, q \leq 1$, generated by $a$ and $b$ satisfying $a^{*} a-q a a^{*}=1-q, \quad b^{*} b-p b b^{*}=1-p, \quad a b=b a, \quad a^{*} b=b a^{*}, \quad\left(1-a a^{*}\right)\left(1-b b^{*}\right)=0$. The *-subalgebra of $\mathcal{O}\left(S_{p q}^{3}\right)$ generated by $a b$ and $b b^{*}$ can be identified with the *-algebra $\mathcal{O}\left(S_{p q}^{2}\right)$ generated by $f_{0}$ and $f_{1}$ satisfying [4] $f_{0}=f_{0}^{*}, \quad f_{1}^{*} f_{1}-q f_{1} f_{1}^{*}=(p-q) f_{0}+1-p, \quad f_{0} f_{1}-p f_{1} f_{0}=$ $(1-p) f_{1}, \quad\left(1-f_{0}\right)\left(f_{1} f_{1}^{*}-f_{0}\right)=0$. The isomorphism is given by $f_{0} \mapsto b b^{*}$ and $f_{1} \mapsto a b$. It follows from [8, Lemma 4.2] that $\mathcal{O}\left(S_{p q}^{2}\right) \subseteq \mathcal{O}\left(S_{p q}^{3}\right)$ is a principal $\mathcal{O}(U(1))$-extension. Moreover, we have

Lemma 2.1 ([8]) Let $u$ be the unitary generator of $\mathcal{O}(U(1))$. The linear map $\ell: \mathcal{O}(U(1)) \rightarrow$ $\mathcal{O}\left(S_{p q}^{3}\right) \otimes \mathcal{O}\left(S_{p q}^{3}\right)$ given on the basis elements $u^{\mu}, \mu \in \mathbb{Z}$, by the formulas

$$
\begin{gathered}
\ell(1)=1 \otimes 1, \quad \ell(u)=a^{*} \otimes a+q b\left(1-a a^{*}\right) \otimes b^{*}, \quad \ell\left(u^{*}\right)=b^{*} \otimes b+p a\left(1-b b^{*}\right) \otimes a^{*}, \\
\ell\left(u^{\mu}\right)=u^{[1]} \ell\left(u^{\mu-1}\right) u^{[2]}, \quad \ell\left(u^{* \mu}\right)=u^{*[1]} \ell\left(u^{*(\mu-1)}\right) u^{*[2]}, \quad \mu>0,
\end{gathered}
$$

defines a strong connection on $\mathcal{O}\left(S_{p q}^{2}\right) \subseteq \mathcal{O}\left(S_{p q}^{3}\right)$.

The one-dimensional corepresentations of $\mathcal{O}(U(1))$ are labelled by integers. Explicitly, we have $\varphi_{\mu}(1)=1 \otimes u^{-\mu}$. Each $\varphi_{\mu}$ yields a finitely generated projective $\mathcal{O}\left(S_{p q}^{2}\right)$-module (quantum line bundle) $\mathcal{O}\left(S_{p q}^{3}\right)_{\mu}:=\operatorname{Hom}^{\mathcal{O}(U(1))}\left(\mathbb{C}_{\varphi_{\mu}}, \mathcal{O}\left(S_{p q}^{3}\right)\right)$. On the other hand, we have the following irreducible bounded $*$-representations of $\mathcal{O}\left(S_{p q}^{2}\right)$ [4, Proposition 19]:

$$
\begin{aligned}
& \rho_{1}\left(f_{0}\right) e_{k}=\left(1-p^{k}\right) e_{k}, \quad \rho_{1}\left(f_{1}\right) e_{k}=\sqrt{1-p^{k+1}} e_{k+1}, \quad k \geq 0 ; \\
& \rho_{2}\left(f_{0}\right) e_{k}=e_{k}, \quad \rho_{2}\left(f_{1}\right) e_{k}=\sqrt{1-q^{k+1}} e_{k+1}, \quad k \geq 0 .
\end{aligned}
$$

Here $\left\{e_{k}\right\}_{k \geq 0}$ is an orthonormal basis of a separable Hilbert space. Moreover, we have

Lemma 2.2 The pair of representations $\left(\rho_{2}, \rho_{1}\right)$ given by (2.3)-(2.4) yields a 1-summable Fredholm module over $\mathcal{O}\left(S_{p q}^{2}\right)$, so that $\operatorname{tr}_{\rho}(f):=\operatorname{Tr}\left(\rho_{2}(f)-\rho_{1}(f)\right)$ defines a trace on $\mathcal{O}\left(S_{p q}^{2}\right)$.

Theorem 2.3 For all $\mu \in \mathbb{Z}$, the pairing between the cyclic 0-cocycle $\operatorname{tr}_{\rho}$ and the $K_{0}$-class of $\mathcal{O}\left(S_{p q}^{3}\right)_{\mu}$ (Chern number) coincides with the winding number $\mu$, i.e., $\left\langle\operatorname{tr}_{\rho},\left[\mathcal{O}\left(S_{p q}^{3}\right)_{\mu}\right]\right\rangle=\mu$.

Proof outline: The pairing of cyclic cohomology and $K$-theory is given by the evaluation of a cyclic cocycle on the image of the Chern character. In our case (see Section 1) it gives $\left\langle\operatorname{tr}_{\rho},\left[\mathcal{O}\left(S_{p q}^{3}\right)_{\mu}\right]\right\rangle=$ $\operatorname{tr}_{\rho}\left(\operatorname{chg}_{0}\left(\varphi_{\mu}\right)\right)=\operatorname{tr}_{\rho}\left(\left(u^{-\mu}\right)^{<2>}\left(u^{-\mu}\right)^{<1>}\right)$. The last expression can be computed explicitly as a function of $p$ or $q$, depending on whether $\mu$ is positive or negative. Surprisingly, these functions can be identified with a certain expression appearing in the index computation carried out in [7]. Since the latter is proven therein to be the constant $\mu$, the assertion of the theorem follows.

The $\operatorname{trace} \operatorname{tr}_{\rho}$ computes the Chern numbers of $\mathcal{O}\left(S_{p q}^{3}\right)_{\mu}$. In order to determine the rank of these modules, we can employ any character of $\mathcal{O}\left(S_{p q}^{3}\right)$. Indeed, let $\delta$ be an algebra homomorphism from $\mathcal{O}\left(S_{p q}^{3}\right)$ to $\mathbb{C}$. (See $[8]$ for the classification of irreducible representations of $\mathcal{O}\left(S_{p q}^{3}\right)$, including one-dimensional ones.) Then $\left\langle\delta,\left[\mathcal{O}\left(S_{p q}^{3}\right)_{\mu}\right]\right\rangle=\delta\left(\left(u^{-\mu}\right)^{<2>}\left(u^{-\mu}\right)^{<1>}\right)=\delta\left(\left(u^{-\mu}\right)^{<1>}\left(u^{-\mu}\right)^{<2>}\right)=$ $\varepsilon\left(u^{-\mu}\right)=1$. The last two equalities follow from the general properties $m \circ \ell=\varepsilon$ (see [2]) and $\varepsilon$ (group-like) $=1$, respectively. The characters always pair integrally with $K_{0}$ (e.g., see [10]). On the other hand, as $\operatorname{tr}_{\rho}$ comes from a 1-summable Fredholm module, its pairing with $K_{0}$ is an index of a Fredholm operator [6, p.60], whence also an integer. Therefore, it follows from the linearity of the pairing that we have a group homomorphism $\left(\delta, \operatorname{tr}_{\rho}\right): K_{0}\left(\mathcal{O}\left(S_{p q}^{2}\right)\right) \rightarrow \mathbb{Z} \oplus \mathbb{Z},[p] \mapsto$ $\left(\langle\delta,[p]\rangle,\left\langle\operatorname{tr}_{\rho},[p]\right\rangle\right)$. The point here is that for any $\mu \in \mathbb{Z}$ there exists a rank one projective module with its Chern number equal to $\mu$. More formally, we have

Corollary 2.4 The image of the positive cone of $K_{0}\left(\mathcal{O}\left(S_{p q}^{2}\right)\right)$ under $\left(\delta, \operatorname{tr}_{\rho}\right): K_{0}\left(\mathcal{O}\left(S_{p q}^{2}\right)\right) \rightarrow \mathbb{Z} \times \mathbb{Z}$ contains $\mathbb{Z}_{+} \times \mathbb{Z}$. 


\section{Hopf fibrations of $S U_{q}(2)$ over generic Podleś spheres}

In this section, we work with the Hopf $*$-algebra $\left.\mathcal{O}\left(S U_{q}(2)\right), q \in\right] 0,1[$, generated by elements $\alpha$ and $\gamma$ satisfying [14] $\alpha \gamma=q \gamma \alpha, \quad \alpha \gamma^{*}=q \gamma^{*} \alpha, \quad \gamma \gamma^{*}=\gamma^{*} \gamma, \quad \alpha^{*} \alpha+\gamma^{*} \gamma=1, \quad \alpha \alpha^{*}+q^{2} \gamma \gamma^{*}=1$, and with the Podleś spheres [13]. A uniform description of all Podleś spheres can be obtained by rescaling generators used in [13]. Then the coordinate $*$-algebras of the quantum spheres $S_{q, s}^{2}, s \in[0,1]$, are defined by generators $K$ and $L$ satisfying the relations $K=K^{*}, \quad L K=q^{2} K L, \quad L^{*} L+K^{2}=$ $\left(1-s^{2}\right) K+s^{2}, \quad L L^{*}+q^{4} K^{2}=\left(1-s^{2}\right) q^{2} K+s^{2}$. We can view $\mathcal{O}\left(S_{q, s}^{2}\right)$ as a subalgebra of $\mathcal{O}\left(S U_{q}(2)\right)$ via the formulas $K=s\left(\gamma \alpha+\alpha^{*} \gamma^{*}\right)+\left(1-s^{2}\right) \gamma^{*} \gamma, \quad L=s\left(\alpha^{2}-q \gamma^{* 2}\right)+\left(1-s^{2}\right) \alpha \gamma^{*}$. Next, let us define the quotient coalgebra $\mathcal{O}\left(S U_{q}(2)\right) / J_{s}$, where $J_{s}$ is the coideal right ideal generated by $K, L-s, L^{*}-s$. One can show that it coincides with $\mathcal{O}(U(1))$ viewed as a coalgebra, and that $\mathcal{O}\left(S_{q, s}^{2}\right)=\mathcal{O}\left(S U_{q}(2)\right)^{c o \mathcal{O}\left(S U_{q}(2)\right) / J_{s}}[1,12]$. Moreover, one can prove that $\mathcal{O}\left(S_{q, s}^{2}\right) \subseteq \mathcal{O}\left(S U_{q}(2)\right)$ is a principal $\mathcal{O}\left(S U_{q}(2)\right) / J_{s}$-extension [3]. With the help of [2], the latter follows from an explicit construction of a strong connection:

Lemma 3.1 ([3]) Let $i: \mathcal{O}(U(1)) \rightarrow \mathcal{O}\left(S U_{q}(2)\right)$ be the linear map defined on the basis elements $u^{\mu}, \mu \in \mathbb{Z}$, by the formulas

$$
\begin{aligned}
& i\left(u^{-\mu}\right):=\left\{\begin{array}{cc}
\prod_{j=0}^{-\mu-1} h_{j} & \text { for } \mu<0 \\
1 & \text { for } \mu=0 \\
\prod_{j=0}^{\mu-1} k_{j} & \text { for } \mu>0,
\end{array} \quad\right. \text { (products increase from left to right) } \\
& h_{j}:=\frac{\alpha+q^{j} s\left(\gamma-q \gamma^{*}\right)+q^{2 j} s^{2} \alpha^{*}}{1+q^{2 j} s^{2}}, \quad k_{j}:=\frac{\alpha^{*}-q^{-j} s\left(\gamma-q \gamma^{*}\right)+q^{-2 j} s^{2} \alpha}{1+q^{-2 j} s^{2}} .
\end{aligned}
$$

Then $\ell=(S \otimes \mathrm{id}) \circ \Delta \circ i$ is a strong connection on $\mathcal{O}\left(S_{q, s}^{2}\right) \subseteq \mathcal{O}\left(S U_{q}(2)\right)$.

Much as before, we associate to every corepresentation $\varphi_{\mu}$ a finitely generated projective left $\mathcal{O}\left(S_{q, s}^{2}\right)$-module $\mathcal{O}\left(S U_{q}(2)\right)_{\mu, s}:=\operatorname{Hom}^{\mathcal{O}\left(S U_{q}(2)\right) / J_{s}}\left(\mathbb{C}_{\varphi_{\mu}}, \mathcal{O}\left(S U_{q}(2)\right)\right)$. On the other hand, using the representations [13]

$$
\begin{gathered}
\pi_{-}(K) e_{n}=-s^{2} q^{2 n} e_{n}, \quad \pi_{-}(L) e_{n}=\lambda_{n}^{-}(q, s) e_{n-1}, \quad \lambda_{n}^{-}(q, s)=s \sqrt{1-\left(1-s^{2}\right) q^{2 n}-s^{2} q^{4 n}} \\
\pi_{+}(K) e_{n}=q^{2 n} e_{n}, \quad \pi_{+}(L) e_{n}=\lambda_{n}^{+}(q, s) e_{n-1}, \quad \lambda_{n}^{+}(q, s)=\sqrt{s^{2}+\left(1-s^{2}\right) q^{2 n}-q^{4 n}}
\end{gathered}
$$

one can prove

Lemma $3.2([\mathbf{1 1}])$ For any $q \in] 0,1\left[, s \in[0,1]\right.$, the pair of representations $\left(\pi_{-}, \pi_{+}\right)$yields a 1-summable Fredholm module over $\mathcal{O}\left(S_{q, s}^{2}\right)$, so that $\operatorname{tr}_{\pi}:=\operatorname{Tr} \circ\left(\pi_{-}-\pi_{+}\right)$is a trace on $\mathcal{O}\left(S_{q, s}^{2}\right)$.

Theorem 3.3 For all $\mu \in \mathbb{Z}$, the pairing between the cyclic 0-cocycle $\operatorname{tr}_{\pi}$ and the $K_{0}$-class of $\mathcal{O}\left(S U_{q}(2)\right)_{\mu, s}$ (Chern number) coincides with the winding number $\mu$, i.e., $\left\langle\operatorname{tr}_{\pi},\left[\mathcal{O}\left(S U_{q}(2)\right)_{\mu, s}\right]\right\rangle=\mu$.

Proof outline: The proof rests on the following three facts: $\left\langle\operatorname{tr}_{\pi},\left[\mathcal{O}\left(S U_{q}(2)\right)_{\mu, s}\right]\right\rangle$ is a rational function of $q$ and $s$, it is an integer, and $\left\langle\operatorname{tr}_{\pi},\left[\mathcal{O}\left(S U_{q}(2)\right)_{\mu, s}\right]\right\rangle(q, 0)=\mu$. The first claim can be proven with the help of the Chern-Galois character [2], the second follows from the noncommutative index formula [6, p.60], and the third has been obtained in [7, Theorem 2.1]. Since an integer-valued rational function on a connected set has to be constant, we can conclude that $\left\langle\operatorname{tr}_{\pi},\left[\mathcal{O}\left(S U_{q}(2)\right)_{\mu, s}\right]\right\rangle(q, s)=\left\langle\operatorname{tr}_{\pi},\left[\mathcal{O}\left(S U_{q}(2)\right)_{\mu, s}\right]\right\rangle(q, 0)=\mu$. 
Corollary 3.4 The image of the positive cone of $K_{0}\left(\mathcal{O}\left(S_{q, s}^{2}\right)\right)$ under $\left(\varepsilon, \operatorname{tr}_{\pi}\right): K_{0}\left(\mathcal{O}\left(S_{q, s}^{2}\right)\right) \rightarrow \mathbb{Z} \times \mathbb{Z}$ contains $\mathbb{Z}_{+} \times \mathbb{Z}$. (Here $\varepsilon$ is the counit of $\mathcal{O}\left(S U_{q}(2)\right)$.)

Acknowledgements. This work has been partially supported by a Marie Curie Fellowship HPMF-CT-200000523 (P.M.H.), Universität Leipzig (P.M.H., W.S.), Deutsche Forschungsgemeinschaft (R.M.), Research Grants Committee of the University of Newcastle and Max-Planck-Institut für Mathematik Leipzig (W.S.). All three authors are grateful to Mathematisches Forschungsinstitut Oberwolfach for support via its Research in Pairs programme.

\section{References}

[1] Brzeziński T., Quantum homogeneous spaces as quantum quotient spaces, J. Math. Phys. 37 (1996) $2388-2399$.

[2] Brzeziński T., Hajac P.M., The Chern-Galois character, joint project (see http://www.fuw.edu.pl/ pmh for a preliminary version).

[3] Brzeziński T., Majid S., Quantum geometry of algebra factorisations and coalgebra bundles, Commun. Math. Phys. 213 (2000) 491-521.

[4] Calow D., Matthes R., Covering and gluing of algebras and differential algebras, J. Geom. Phys. 32 (2000) 364-396.

[5] Calow D., Matthes R., Connections on locally trivial quantum principal fibre bundles, J. Geom. Phys. 41 (2002) 114-165.

[6] Connes A., Noncommutative differential geometry, Inst. Hautes Études Sci. Publ. Math. 62 (1985) 257-360.

[7] Hajac P.M., Bundles over quantum sphere and noncommutative index theorem, K-Theory 21 (2000) 141-150.

[8] Hajac P.M., Matthes R., Szymański W., A locally trivial quantum Hopf fibration, to appear in Algebr. Represent. Theory, math.QA/0112317.

[9] Klimek S., Lesniewski A., A two-parameter quantum deformation of the unit disc, J. Funct. Anal. 115 (1993) $1-23$.

[10] Loday J.-L., Cyclic homology, Springer-Verlag, Berlin, 1998.

[11] Masuda T., Nakagami Y., Watanabe J., Noncommutative differential geometry on the quantum two sphere of Podleś. I: An algebraic viewpoint, $K$-Theory 5 (1991) 151-175

[12] Müller E.F., Schneider H.-J., Quantum homogeneous spaces with faithfully flat module structures, Israel J. Math. 111 (1999) 157-190.

[13] Podleś P., Quantum spheres, Lett. Math. Phys. 14 (1987) 193-202.

[14] Woronowicz S.L., Twisted $S U(2)$ group. An example of a non-commutative differential calculus, Publ. RIMS, Kyoto Univ. 23 (1987) 117-181. 